\title{
Análisis del factor de impacto de las revistas científicas latinoamericanas
}

\author{
José F Téllez-Zentenoํㅜ, Luis E Morales-Buenrostro², \\ Bruno Estañol ${ }^{1}$. \\ Impact factor of \\ Latin American medical journals
}

\begin{abstract}
Background: Latin American medical journals have a low impact factor. Higher quality articles originated in Latin American countries are published in North American or European journals. Aim: To analyze the impact factor of Latin-American journals according to the language of publication. Material and methods: The data base of periodic journals of the Thomson ISI (Journal of Citation Report) in the year 2004 was used for the analysis. Four countries with more than one journal in the data base of the Thomson ISI were included (Argentina, Brazil, Chile and Mexico). Results: Few Latin-American journals are included in the Thomson ISI data base. The mean impact factor was 0.76 (0.23-3.2) for eight Mexican journals, $0.66(0.10-2.1)$ for eight Chilean journals, 0.39 (0.06-0.7) for five Argentinian journals and 0.41 (0.09-1.1) for 16 Brazilian journals. The mean impact factor for 11 journals written in English was 0.74 (0.12-2.1), 0.53 (0.09-3.2) for 18 bilingual journals and 0.28 (0.06-0.56) for eight journals written in native language. The differences between countries and languages were not statistically significant. Conclusions: The journal impact factor was similar in the four countries studied. A non-significant higher impact factor was observed in Latin-American journals published in English (Rev Méd Chile 2007; 135: 480-7).
\end{abstract}

(Key words: Databases, bibliographic; Journalism, medical; Language)

Recibido el 17 de enero de 2006. Aceptado el 10 de octubre, 2006.

${ }^{1}$ Departamento de Neurología y Psiquiatría del Instituto Nacional de Ciencias Médicas y Nutrición «Salvador Zubirán». 2Departamento de Nefrología del Instituto Nacional de Ciencias Médicas y Nutrición «Salvador Zubirán». Ciudad de México, México.

$\mathrm{E}^{\mathrm{n}}$ los últimos años ha crecido la preocupación por el futuro de las revistas científicas latinoamericanas. Los mejores trabajos de los investigadores de esta región se publican en revistas extranjeras ya que las revistas latinoamericanas tienen bajo sactor de impacto». Las revistas médi-

Correspondencia a: Dr. José Francisco Téllez-Zenteno. Division of Neurology. Department of Medicine. Royal University Hospital 103 Hospital Drive. Box 26, Room 1622. Saskatoon SK S7N OW8 Canada. Tel (306)9668011. Fax: (306)9668008. E mail: jft084@mail.usask.ca cas en los países de Latinoamérica son abundantes, pero de poca calidad, teniendo como principal problema la falta de publicación de trabajos originales de alta calidad que sean potencialmente susceptibles de ser citados en otras revistas internacionales ${ }^{1}$.

El sactor de impacto»lo determina el Instituto de Información Científica (ISI-siglas en inglésllamado actualmente Thomson ISI). El Thomson ISI fue fundado en 1958, con la función de dar a los investigadores un acceso a información relacionada con la investigación científica de alta 
calidad. El Thomson ISI cubre más de 16.000 revistas en diferentes áreas relacionadas con las ciencias y publica, anualmente, una lista con el factor de impacto de cada una de las revistas incluidas en sus archivos ${ }^{2-4}$. El factor de impacto es una medida aceptada universalmente y su principal uso se encuentra en la evaluación y calificación de las revistas periódicas de acuerdo a su número de $\operatorname{citas}^{2}$.

Un aspecto fuertemente relacionado con la difusión de las investigaciones publicadas en las revistas latinoamericanas es el idioma de publicación. En los últimos años, la transformación al idioma inglés de revistas provenientes de países donde el idioma inglés no es el oficial, en la búsqueda de mejorar su difusión, ha sido numerosa. Por citar algunos ejemplos, prácticamente todas las revistas biomédicas europeas importantes se encuentran ya escritas en inglés y en Asia, la mayoría de las revistas biomédicas se encuentra en el proceso de conversión al inglés. Esta transformación en Latinoamérica y en España ha comenzado lentamente $y$, al parecer, existe menor aceptación que en otras áreas geográficas hacia este cambio, ya que se considera que el papel primordial de estas revistas es la difusión de la información médica en los propios países de publicación de habla castellana o portuguesa. Esta razón y otras han sido expuestas por los comités editoriales de las revistas latinoamericanas ${ }^{1,5}$.

Considerando la escasa información disponible sobre el factor de impacto de las revistas latinoamericanas, realizamos un análisis con el fin de conocer el estado actual. Los objetivos de este estudio son los siguientes: 1) Analizar el factor de impacto de las revistas latinoamericanas, 2) Evaluar el idioma de publicación y su relación con el factor de impacto, y 3) Discutir perspectivas a futuro para las revistas latinoamericanas.

\section{Material y MÉTOdo}

Para analizar el factor de impacto de las revistas latinoamericanas, se utilizaron los datos contenidos en la base de datos de revistas periódicas del Thomson ISI (Journal of Citation Report) ${ }^{6}$. El factor de impacto es el número de citas obtenidas por la revista en un año, dividido por el número de manuscritos publicados en los dos años pre- vios. Para fines de este estudio, el factor de impacto correspondiente a 2004 es expresado con la siguiente fórmula:

$$
\begin{aligned}
& \text { \# Citas obtenidas en } 2004 \text { de los artículos } \\
& \text { publicados en } 2002 \text { y } 2003 \\
& \hline \text { \# Artículos publicados en } 2002 \text { y } 2003
\end{aligned}
$$

La base de datos Thomson ISI permite el cálculo del factor de impacto a lo largo del año, sin embargo, para este estudio se utilizó la cifra final de 2004. La base de datos Thomson ISI permite analizar el número de revistas por país, el factor de impacto de cada revista, el tópico de cada revista y el idioma en que se publica ${ }^{6}$.

Se seleccionaron cuatro países de Latinoamérica que cuentan con más de una revista en el Thomson ISI, para que los datos tuvieran mayor representatividad (Argentina, Brasil, Chile y México). Cabe destacar que los demás países latinoamericanos cuentan con ninguna o sólo una revista en la base de datos Thomson ISI, la que incluye revistas en todas las áreas del conocimiento.

Para el análisis, se utilizó estadística descriptiva de acuerdo al nivel de medición de las variables. Se utilizó la prueba de análisis de varianza de rangos para comparar el factor de impacto en algunos grupos de interés. Los datos fueron analizados en la versión 10 de SPSS. Un valor de $\mathrm{p}<0,05$ fue considerado estadísticamente significativo.

\section{RESULTADOS}

Revistas latinoamericanas incluidas en la base de datos Thomson ISI de los países seleccionados (Tablas 1 a 4).

Argentina (Tabla 1). Cuenta con 5 revistas registradas, con factores de impacto que van de 0,06 a 0,70. Dos revistas son bilingües, dos redactadas en español y una en inglés. Tres revistas pertenecen al área biomédica y las otras dos a disciplinas diversas.

Chile (Tabla 2). Cuenta con 8 revistas registradas con factores de impacto que van del 0,10 a 2,1. Tres revistas están escritas en inglés, dos en español y tres son bilingües. Dos revistas son del área biomédica y seis son de disciplinas diversas.

Brasil (Tabla 3). Cuenta con 16 revistas registradas con factores de impacto entre 0,09 y 1,1. Seis se encuentran redactadas en el idioma inglés, 
Tabla 1. Revistas científicas argentinas registradas en la base de datos Thomson ISI en $2004, n=5$

\begin{tabular}{|lccc|}
\hline Revista & Idioma & Factor de impacto & Temática \\
\hline Ameghiniana & Bilingüe & 0,70 & Paleontología \\
Biocell & Español & 0,56 & Biología celular \\
Medicina Buenos Aires & Bilingüe & 0,32 & Medicina General/Medicina Interna \\
Latin American Applied Research & Inglés & 0,31 & Ingeniería, Química \\
Acta Bioquímica Clínica Latinoaméricana & Español & 0,06 & Bioquímica \\
\hline
\end{tabular}

Tabla 2. Revistas científicas chilenas registradas en la base de datos Thomson ISI en $2004, n=8$

\begin{tabular}{|c|c|c|c|}
\hline Revista & Idioma & Factor de impacto & Temática \\
\hline Biological Research & Inglés & 2,1 & Biología \\
\hline Revista Geológica de Chile & Bilingüe & 0,91 & Geología \\
\hline $\begin{array}{l}\text { Revista Chilena de } \\
\text { Historia Natural }\end{array}$ & Bilingüe & 0,58 & Biodiversidad, ecología \\
\hline $\begin{array}{l}\text { Boletín de la Sociedad } \\
\text { Chilena de Química }\end{array}$ & Bilingüe & 0,30 & Química \\
\hline $\begin{array}{l}\text { Revista Médica de Chile } \\
\text { Archivos de Medicina }\end{array}$ & Español & 0,27 & Medicina General/Medicina Interna \\
\hline $\begin{array}{l}\text { Veterinaria } \\
\text { Electronic Journal of }\end{array}$ & Español & 0,10 & Veterinaria \\
\hline Biotechnology & Inglés & 0,72 & $\begin{array}{l}\text { Biotechnology/applied } \\
\text { microbiology }\end{array}$ \\
\hline $\begin{array}{l}\text { Journal of the Chilean } \\
\text { Chemical Society }\end{array}$ & Inglés & 0,38 & Chemistry \\
\hline
\end{tabular}

8 son bilingües y 2 en portugués. Cinco revistas son del área biomédica y el resto pertenecen a diversas disciplinas.

México (Tabla 4). Cuenta con 8 revistas registradas con un factor de impacto entre 0,22 y 3,2 . Una de ellas se encuentra en inglés, 1 en español y las 6 restantes son bilingües. Dos revistas son del área biomédica y el resto pertenece a diversas disciplinas.

Análisis por país (Tabla 5). El porcentaje de revistas escritas en el idioma inglés o combinación (inglés + nacional) en los cuatro países es mayor a $60 \%$. No se observaron diferencias estadísticamente significativas entre el factor de impacto de los 4 países evaluados $(\mathrm{p}<0,05)$.

Revistas biomédicas con mayor factor de impacto en Latinoamérica de acuerdo a la base de datos Thomson ISI en 2004. Las 5 revistas biomédicas con mayor factor de impacto se pueden observar en la Tabla 6. La revista con mayor factor impacto en 2004 fue Archives of Medical Research con 1,28. El segundo y tercer lugar lo ocupan 2 revistas brasileñas escritas en inglés (Brazilian Journal of Medical and Biological Research y Memorias do Instituto Oswaldo Cruz).

En la Tabla 7 podemos observar el factor de impacto de las revistas de acuerdo al idioma en que están escritas. No se observaron diferencias estadísticamente significativas entre los idiomas en que están escritas las revistas $(p>0,05)$, sin embargo, se observó una tendencia a tener un factor de impacto más alto en las revistas bilingües y en las escritas completamente en inglés. 
Tabla 3. Revistas científicas brasileñas registradas en la base de datos T homson ISI en $2004, n=16$

\begin{tabular}{|c|c|c|c|}
\hline Revista & Idioma & Factor de impacto & Temática \\
\hline \multicolumn{4}{|l|}{ Journal of the Brazilian } \\
\hline Chemical Society & Inglés & 1,1 & Química \\
\hline \multicolumn{4}{|l|}{ Brazilian Journal of Medical } \\
\hline and Biological Research & Inglés & 0,82 & $\begin{array}{l}\text { Medicina, medicina } \\
\text { experimental }\end{array}$ \\
\hline Brazilian Journal of Physics & Inglés & 0,43 & Física \\
\hline Memorias do Instituto Oswaldo Cruz & Inglés & 0,74 & $\begin{array}{c}\text { Parasitología, medicina } \\
\text { tropical }\end{array}$ \\
\hline Química Nova & Bilingüe & 0,62 & Química \\
\hline \multicolumn{4}{|l|}{ Anais da Academia Brasileira de } \\
\hline Ciencias & Bilingüe & 0,43 & Ciencias (multidisciplinaria) \\
\hline Revista Brasileria de Zootecnia & Bilingüe & 0,25 & $\begin{array}{l}\text { Agricultura, veterinaria, } \\
\text { ciencias de los animales }\end{array}$ \\
\hline Pesquisa Veterinaria Brasileira & Bilingüe & 0,37 & Veterinaria \\
\hline Genetics and Molecular Biology & Bilingüe & 0,31 & $\begin{array}{l}\text { Bioquímica, biología } \\
\text { molecular, genética }\end{array}$ \\
\hline Arquivos de Neuro-psiquiatria & Portugués & 0,40 & $\begin{array}{l}\text { Neurociencias } \\
\text { Psiquiatría }\end{array}$ \\
\hline \multicolumn{4}{|l|}{ Brazilian Journal of Chemical } \\
\hline \multicolumn{4}{|l|}{ Boletim da Sociedad Brasileira } \\
\hline de Matematica & Bilingüe & 0,23 & Matemáticas \\
\hline \multicolumn{4}{|l|}{ Brazilian Archives of Biology } \\
\hline Brazilian Journal of Microbiology & Inglés & 0,12 & Microbiología \\
\hline Pesquisa Agropecuaria Brasileira & Portugués & 0,37 & Agricultura \\
\hline \multicolumn{4}{|l|}{ Arquivo Brasileiro de Medicina } \\
\hline velemilland e Loolechind & Dillingue & 0,09 & velemindand \\
\hline
\end{tabular}

Tabla 4. Revistas científicas mexicanas registradas en la base de datos T homson ISI en $2004, n=8$

\begin{tabular}{|lccc|}
\hline Revista & Idioma & Factor de impacto & Temática \\
\hline Revista Mexicana de & Bilingüe & 3,2 & Astronomía, astrofísica \\
Astronomía y Astrofísica & Inglés & 1,28 & Medicina, medicina experimental \\
Archives Medical Research & Bilingüe & 0,39 & Biología marina \\
Ciencias Marinas & & & Medicina interna/medicina general \\
Revista de Investigación & Bilingüe & 0,27 & Meteorología, ciencias de la atmósfera \\
Clínica & Bilingüe & 0,28 & Física \\
Atmósfera & Bilingüe & 0,22 & Matemáticas \\
Revista Mexicana de Física & Ingeniería civil \\
Boletín de la Sociedad & Bilingüe & 0,29 & \\
Matemática Mexicana & & 0,23 & Español \\
Ingeniería Hidráulica en & & & \\
México & &
\end{tabular}


Tabla 5. N úmero de revistas científicas por país, porcentaje de revistas en un idioma diferente al nacional y factor de impacto por país de acuerdo a la base de datos T homson ISI en 2004, n =37

\begin{tabular}{|lccc|}
\hline País & $\begin{array}{c}\text { No de revistas } \\
\text { en el ISI }\end{array}$ & $\begin{array}{c}\text { Número y porcentaje de } \\
\text { revistas bilingües (inglés }+ \\
\text { nacional) o en inglés }\end{array}$ & $\begin{array}{c}\text { Factor de impacto } \\
\text { (promedio-intervalos) }\end{array}$ \\
\hline México & 8 & $7(88 \%)$ & $0,76(0,23-3,2)$ \\
Chile & 8 & $6(75 \%)$ & $0,66(0,10-2,1)$ \\
Argentina & 5 & $3(60 \%)$ & $0,39(0,06-0,7)$ \\
Brasil & 16 & $14(88 \%)$ & $0,41(0,09-1,1)$ \\
Total & 37 & $30(81 \%)$ & $0,55(0,23-2,1)$ \\
\hline
\end{tabular}

No se encontraron diferencias estadísticas entre los 4 países $(p<0,05)$.

Tabla 6. Las 5 revistas biomédicas con mayor factor de impacto en Latinoamérica de acuerdo a la base de datos T homson ISI en 2004

\begin{tabular}{|c|c|c|c|c|}
\hline Revista & Idioma & $\begin{array}{l}\text { Factor de } \\
\text { Impacto }\end{array}$ & Temática & País \\
\hline Archives of Medical Research & Inglés & 1,28 & $\begin{array}{l}\text { Medicina, medicina } \\
\text { expenimental }\end{array}$ & México \\
\hline $\begin{array}{l}\text { Brazilian Journal of Medical } \\
\text { and Biological Research }\end{array}$ & Inglés & 0,82 & $\begin{array}{l}\text { Medicina, medicina } \\
\text { experimental }\end{array}$ & Brasil \\
\hline \multicolumn{5}{|l|}{ Memorias do Instituto } \\
\hline Oswaldo Cruz & Inglés & 0,74 & $\begin{array}{l}\text { Parasitología, } \\
\text { medicina tropical }\end{array}$ & Brasil \\
\hline Medicina Buenos Aires & Bilingüe & 0,32 & $\begin{array}{l}\text { Medicina general/ } \\
\text { medicina interna }\end{array}$ & Argentina \\
\hline \multicolumn{5}{|l|}{ Revista de } \\
\hline Investigación Clínica & Bilingüe & 0,27 & $\begin{array}{l}\text { Medicina intema/ } \\
\text { medicina general }\end{array}$ & México \\
\hline
\end{tabular}

Tabla 7. Factor de impacto de acuerdo al idioma en que están escritas las revistas biomédicas latinoamericanas, de acuerdo a la base de datos T homson ISI en 2004, n =37

\begin{tabular}{|lcc|}
\hline Idioma de la revista & $\begin{array}{l}\mathrm{n}=35 \\
\mathrm{n}(\%)\end{array}$ & $\begin{array}{c}\text { Factor de impacto } \\
\text { (promedio-intervalos) }\end{array}$ \\
\hline Idioma nacional & $6(16 \%)$ & $0,28(0,06-0,56)$ \\
Bilingüe (idioma nacional + inglés) & $20(54 \%)$ & $0,53(0,09-3,2)$ \\
Inglés & $11(31 \%)$ & $0,74(0,12-2,1)$ \\
\hline
\end{tabular}

No se encontraron diferencias estadísticas entre los 3 grupos $(\mathrm{p}<0,05)$. 


\section{DiSCUSIÓN}

De nuestro análisis podemos considerar algunos aspectos interesantes: por una parte, los cuatro países analizados tienen pocas revistas registradas en el Thomson ISI, particularmente si los comparamos con países como Inglaterra y Estados Unidos que tienen más de 1.000 y 2.000 revistas respectivamente ${ }^{6}$. Por otra parte, el factor de impacto es muy bajo en la mayoría de las revistas latinoamericanas, si consideramos que el factor de impacto de algunas de las revistas biomédicas más prestigiadas en el mundo es mayor de 50. La única revista biomédica latinoamericana que tuvo un factor de impacto mayor a 1, en 2004, fue Archives of Medical Research (AMR), que se encuentra totalmente escrita en inglés (Tabla 6). De forma global, podemos observar que el factor de impacto es bajo en los cuatro países (Tabla 5), sin encontrarse diferencias estadísticamente significativas entre ellos salvo una tendencia a tener mayor factor de impacto las revistas publicadas en inglés.

Es claro que el factor de impacto de las revistas latinoamericanas es bajo y esto podría considerarse una mala noticia para los editores de las revistas latinoamericanas. Sin embargo, la mayoría de los editores regionales pasan a un segundo término el factor de impacto y defienden el papel de cada una de estas revistas como uno de los pocos o el único medio de difusión del conocimiento médico en sus propios países ${ }^{7,8}$. Aunque este último argumento sea válido, la situación es crítica para la ciencia latinoamericana y obliga a plantear algunos intermogantes. Creemos que algunos países de Latinoamérica empiezan a apoyar la posibilidad de la conversión al inglés de diversas revistas, para mejorar la difusión internacional de los trabajos de investigación latinoamericanos ${ }^{1,9,10}$.

Acerca del idioma inglés, podemos observar en las primeras cuatro Tablas cómo la mayonía de las revistas latinoamericanas ha cambiado su idioma oficial o se encuentran en vías de cambio. Aunque existe resistencia por el cambio al idioma inglés por los comités de las revistas de países latinoamericanos, la mayonía de las revistas reciben artículos en inglés y muchas están escritas totalmente en inglés. La tendencia es que cada vez sean menos las revistas publicadas en el idioma nacional (Tabla 5). Podemos notar que las revistas con mayor impacto en cada país se encuentran escritas en inglés o en una combinación del idioma nacional y el inglés. En el caso de las revistas biomédicas latinoamenicanas, las que cuentan con mejor factor de impacto también se encuentran escritas en inglés (Tabla 6). Finalmente, el factor de impacto aparece ligeramente mayor en las revistas que están escritas totalmente en inglés que en las que conservan el idioma nacional, aunque la diferencia no es estadísticamente significativa (Tabla 7).

Es evidente que la conversión al inglés es lenta y difícil para todos los países de Latinoaménica, pero es probable que en los próximos años la mayonía de estas revistas serán escritas totalmente en inglés para poder sobrevivir y competir con revistas de calidad internacional ${ }^{1,9,11}$. Aunque no hay una evidencia estadística, hasta ahora, de que la conversión al inglés de los artículos mejora el factor de impacto, sí se nota una clara tendencia a este respecto. Este efecto tendrá que ser evaluado a mediano y a largo plazo para evaluar su potencial uso. De acuerdo a los datos del Instituto Pasteur, la conversión de sus revistas al inglés no ha sido benéfica, ya que no ha mejorado su factor de impacto ${ }^{5}$. Sin embargo, en otros países, como Alemania, se observa que el factor de impacto ha aumentado significativamente y en este país se recomienda la conversión al idioma inglés ${ }^{5,12,13}$. Finalmente, algunos países, como España y México, defienden el idioma español, ya que consideran que la difusión en los países de origen es más importante, restándole importancia a la difusión intemacional ${ }^{8,14-}$ 18. Para valorar si existe algún cambio en las revistas latinoamericanas habrá que esperar algunos años para ver los resultados, sin embargo la tendencia actual muestra que la conversión será el camino que tomen la mayoná de las revistas.

La conversión al idioma inglés no es una situación particular de los países de Latinoamérica y no sólo está relacionada con el factor de impacto sino con la difusión de los artículos de investigación. En los últimos años se ha adoptado rápidamente en el ámbito internacional al idioma inglés como el idioma científico, disminuyendo la difusión internacional de información escrita en otros idiomas. Un ejemplo de este problema es el citado por Macias-Hernández en una revista mexicana hace algunos años. El autor describió 10 casos de infección sistémica por aeromonas en México, en $1988^{9}$. Posteriormente, el autor hizo mención de subsecuentes reportes de casos por aeromonas descritos en la literatura internacional donde su artículo nunca fue citado. Finalmente, 
en 1993 Kelly y cols ${ }^{19}$ describieron la serie más grande publicada hasta ese entonces, con 56 casos, en la cual no se citó el artículo publicado por Macias-Hernández. De hecho, Kelly ${ }^{19}$ describió que el espectro clínico en países tropicales es desconocido, siendo que los casos descritos por Macias muestran una presentación clínica diferente. La falta de reconocimiento de este artículo y muchos más, probablemente no se debe a la falta de difusión, ya que los resúmenes aparecen en índices internacionales, sino probablemente al idioma en el que está escrito. Es probable que los investigadores de otros países, cuando ven que los artículos se encuentran escritos en español, no se toman el trabajo de leerlos. Por otra parte, artículos de temas propios de Latinoamérica, como cisticercosis, escritos en revistas en inglés alcanzan altos grados de impacto y difusión ${ }^{20}$. Considerando lo anterior, la conversión al idioma inglés sería una necesidad si realmente deseamos mejorar el impacto y la difusión de investigaciones publicadas en revistas latinoamericanas.

Un buen ejemplo de la conversión al idioma inglés y su relación con el factor de impacto es el caso de las dos revistas biomédicas mexicanas (Revista de Investigación Clínica-RIC y Archives of Medical Research-AMR). La RIC es una de las revistas biomédicas con mayor tradición en Latinoamérica, escrita en su mayoría en idioma castellano y que ha mantenido su periodicidad y calidad a lo largo de muchos años. La revista ha incursionado con una página electrónica en el sitio de IMBIOMED y ha realizado algunos cambios en su estructura para hacerla más atractiva ${ }^{8,21}$. El AMR es una revista recientemente convertida al inglés y relacionada con una de las compañías que difunden libros y revistas científicas con mayor importancia en el mundo (compañía editora Elsevier) y, por lo tanto, cuenta con muy buena difusión y disponibilidad de sus artículos en texto completo a nivel internacional. Esta revista ha tomado un carácter internacional en los últimos años, además de que su factor de impacto ha aumentado considerablemente a partir de su conversión al inglés, en contraste con la RIC, que continúa con el mismo factor de impacto desde hace muchos años ${ }^{22,23}$. Debido a su creciente factor de impacto, recientemente AMR ha sido distinguida como la revista líder en Latinoamérica en investigación biomédica, destacando la calidad de sus artículos y su poten- cial futuro 23,24 . La revista AMR es un buen ejemplo de lo que podría pasar con algunas revistas latinoamericanas en el futuro para incrementar la difusión internacional de las investigaciones realizadas en Latinoamérica.

Acerca de la difusión en los países de publicación, el caso de algunas revistas biomédicas latinoamericanas como la RIC, Medicina de Buenos Aires y la Revista Médica de Chile son excelentes ejemplos de revistas médicas consideradas de alta calidad en sus respectivos países ${ }^{7,16}$. Algunas de ellas son la principal fuente de educación médica para muchos estudiantes en sus propios países, representan el primer contacto con la investigación para muchos médicos y constituyen la primera experiencia para publicar sus trabajos para sus investigadores clínicos ${ }^{7,8,21}$. Además de que son foros ideales para publicar experiencias de enfermedades que son propias de los países en cuestión y que tal vez no serían útiles en otros países. Por estas razones, los cuerpos editoriales hacen a un lado el factor de impacto y defienden el papel de difusión de la información que tienen estas revistas en sus respectivos países ${ }^{8,16,25}$. Es claro también que el factor de impacto fue creado por países del primer mundo, con el propósito de promover y vender más una revista diciendo que tiene un alto impacto y, por lo tanto, mejor calidad. Sin embargo, hay que reconocer que es uno de los pocos índices reconocidos internacionalmente ${ }^{2-4,26}$.

Aunque el factor de impacto sea una medida injusta para diversos países, incluyendo los latinoamericanos, es una medición aceptada universalmente que debemos tomar en cuenta cada día más. Algunas medidas potenciales para aumentar la difusión internacional de las revistas latinoamericanas incluyen su publicación en dos idiomas: una versión local en el idioma nacional, que cumpliría con la labor de difusión en sus respectivos países, y una segunda versión publicada en inglés para difundirla internacionalmente y tener la oportunidad de que las investigaciones sean citadas y reconocidas en otras partes del mundo. Estas opciones son onerosas a primera vista. Otro paso que en el futuro debe ser considerado, es la posible difusión de las revistas latinoamericanas por empresas internacionales encargadas de difundir ciencia, permitiendo una amplia disponibilidad de los artículos en texto completo. Esto último ha sido implementado por la revista $\mathrm{AMR}$ 
en México, con Elsevier, obteniendo gran difusión de sus artículos de investigación ${ }^{24}$. En general, es claro que el bajo factor de impacto de las revistas latinoamericanas requiere para su incremento de nuevas y creativas estrategias por parte de los

\section{REFERENCIAS}

1. Pérez-Padila R. [The uncertain future of Mexican medical journals]. Rev Invest Clin 1995; 47: 165-7.

2. BenITEZ-BribIESCA L [The impact factor of medical journals: its use and misuse]. Arch Med Res 1999; 30: 161-2.

3. Coelho PM, Antunes CM, Costa HM, Kroon EG, SOUSA Lima MC, LINARDI PM. The use and misuse of the impact factor» as a parameter for evaluation of scientific publication quality: a proposal to rationalize its application. Braz J Med Biol Res 2003; 36: 1605-12.

4. ElLARD JH. The «mnipotent» Science Citation Index impact factor. Med J Aust 2003; 179: 120.

5. Bracho-Riquelme RL, Pescador-Salas N, Reyes-RomeRO MA. [Bibliometric repercussions of adopting English as the sole language for publication]. Rev Invest Clin 1997; 49: 369-72.

6. Thomson ISI. http://go5.isiknowledge.com.proxy.lib.uwo.ca:2048/portal.cgi. 2004.

7. Goic A. [The Revista Medica the Chile and medical education]. Rev Méd Chile 2002; 130: 719-22.

8. LORIA A, LISKER R. [Objectives, strategies, and problems of the Revista de Investigación Clínica]. Rev Invest Clin 1995; 47: 89-93.

9. MaCiAs-HeRnández AE. [English articles in Revista Investigación Clínica]. Rev Invest Clin 1994; 46: 335-7.

10. Bunout D, Reyes H. [Biomedical papers written by Chilean authors published in international journals in 1997. A review of MEDLINE]. Rev Méd Chile 1998; 126: 677-88.

11. Reyes H, Kauffmann R, Andresen M. [Tradition and progress: now also a bilingual journal?]. Rev Méd Chile 2000; 128: 1187-9.

12. Winkmann G, Schlutius S, Schweim HG. Citation rates of medical German-language journals in English-language papers-do they correlate with the impact factor, and who cites? Klin Monatsbl Augenheilkd 2002; 219: 72-8.

13. Winkmann G, Schlutius S, Schweim HG. Publication languages of Impact Factor journals and of editores hacia el futuro a fin de mejorar la difusión de las investigaciones latinoamericanas y de entrar de una forma más directa a una comunidad científica globalizada que ha adoptado el idioma inglés como la łengua franca»contemporánea.

medical bibliographic databanks. Dtsch Med Wochenschr 2002; 127: 131-7.

14. No Authors Listed. [Scientific articles in Spanish. Editorial board of the Revista de Investigacion Clinica). Rev Invest Clin 1994; 46: 167-9.

15. Aleixandre BR, Valderrama-Zurian JC, Casteliano GM, Simo MR, NavarRo MC. [Archivos de Bronconeumología: Among the 3 Spanish Medical Journals With the Highest National Impact Factors]. Arch Bronconeumol 2004; 40: 563-9.

16. LORIA A, LISKER R. [Brief analysis of the RRevista de Investigación Clínica». Rev Invest Clin 1993; 45: 433-7.

17. MÉndez-Sánchez N, Lazcano PE. [More on Mexican medical journals]. Rev Invest Clin 1995; 47: 507-8.

18. MuriLo-Godinez G. [The difficulties of scientific journals in Spanish]. Rev Invest Clin 1998; 50: 177.

19. KeLu KA, KoehleR JM, Ashdown LR. Spectrum of extraintestinal disease due to Aeromonas species in tropical Queensland, Australia. Clin Infect Dis 1993; 16: 574-9.

20. García HH, Pretell ej, Gilman RH, Martínez SM, Moulton LH, Del Brutto OH et al. A trial of antiparasitic treatment to reduce the rate of seizures due to cerebral cysticercosis. $\mathrm{N}$ Engl J Med 2004; 350: 249-58.

21. GamBa G. [«a Revista de Investigación Clínica» facing new challenges and old conflicts]. Rev Invest Clin 2000; 52: 7-9.

22. Benitez-Bribiesca L. The ups and downs of the impact factor: the case of Archives of Medical Research. Arch Med Res 2002; 33: 91-4.

23. GibBs WW. Lost science in the Third World. Sci Am 1995; 273: 92-9.

24. Benitez-BRiBIeSCA L. Starting a global scientific journal. Science 2004; 304: 1595.

25. LORIA A, LSKER R. [The impact factor of the Revista de Investigacion Clinica]. Rev Invest Clin 1995; 47: 427-31.

26. KURMIS AP. Understanding the limitations of the journal impact factor. J Bone Joint Surg Am 2003; 85-A: 2449-54. 